\title{
CORRELATION OF TEST ROBOTIZATION FRAMEWORKS TO PROPOSE A PROTECTED AND SOLID TECHNIQUE
}

\author{
B.K. SRINIVAS ${ }^{1} \&$ KAVYA $S^{2}$ \\ ${ }^{1}$ Assistant Professor, ISE Department, $R$ V College of Engineering, Bengaluru - 560059, India \\ ${ }^{2} M$. Tech in Software Engineering, ISE Department, $R$ V College of Engineering, Bengaluru - 560059, India
}

\begin{abstract}
The procedure of programming testing points not just at discovering deficiencies in the current programming yet in addition at discovering measures to improve the product as far as effectiveness, exactness and ease of use. It predominantly targets estimating determination, usefulness and execution of a product program or application. It is either done manually or using automated tools. Robot Framework is a Python-based, extensible catchphrase driven computerization system for acknowledgment testing, acknowledgment test driven turn of events, conduct driven turn of events and mechanical procedure mechanization. Automation is done using some of the automation frameworks like Selenium, Cucumber and Selenium with combination of TestNG framework. When compared with these frameworks Robot Framework stands out among above mentioned frameworks because of its unique features. Robot Framework generates results in easy to read format that to it generates both reports and logs in HTML format. This paper presents a comparison of test automation frameworks which is widely used in industry for automation testing and proposing a secure and reliable framework.

KEYWORDS: Selenium; Acceptance; Keyword-Driven; Robot Framework; Automated Testing
\end{abstract}

Received: Jun 01, 2020; Accepted: Jun 20, 2020; Published: Jul 22, 2020 Paper Id.: IJMPERDJUN2020416

\section{INTRODUCTION}

Programming testing is characterized as a movement to check whether the real outcomes coordinate the normal outcomes and to guarantee that the product framework is without imperfection. It includes execution of a product part or framework segment to assess at least one properties. Programming testing likewise assists with distinguishing mistakes, holes or missing prerequisites in opposition to the real necessities. It tends to be either done physically or utilizing computerized apparatuses or it can expressed as the way toward confirming and approving that a product or application is without bug, meets the specialized prerequisites as guided by its structure and advancement and meets the client necessities successfully and proficiently with dealing with all the excellent and limit cases.

The procedure of programming testing points not just at discovering shortcomings in the current programming yet in addition at discovering measures to improve the product as far as productivity, exactness and ease of use. It predominantly targets estimating particular, usefulness and execution of a product program or application.

A computerized testing instrument can playback pre-recorded and predefined activities, contrast the outcomes with the normal conduct and report the achievement or disappointment of these manual tests to a test engineer. 


\section{Types of Software Testing:}

There are many numbers of tests present in software or in software testing which starts from unit testing and ends in system testing. Normally testing is ordered into three classifications:

- Functional Testing.

- Non - Functional Testing.

- Maintenance Testing.

Functional Testing: Utilitarian testing is a sort of programming testing where by the framework is tried against the practical necessities/details. Capacities (or highlights) are tried by taking care of them input and inspecting the yield. Utilitarian testing guarantees that the necessities are appropriately fulfilled by the application.

Non Functional Testing: Non-useful testing is the trying of a product application or framework for its nonutilitarian necessities: the manner in which a framework works, instead of explicit practices of that framework. For instance, programming execution is a wide term that incorporates numerous particular prerequisites like dependability and adaptability.

Maintenance Testing: Upkeep testing is a test that is performed to either recognize hardware issues, analyze gear issues or to affirm that fix measures have been successful. It very well may be performed at either the framework level, the hardware level, or the part level.

\section{OVERVIEW ON AUTOMATION TESTING}

Computerization testing is a Software testing procedure to test and contrast the real result and the normal result. This can be accomplished by composing test contents or utilizing any mechanization testing device. Test mechanization is utilized to robotize dull assignments and other testing undertakings which are hard to perform physically.

Test Automation programming is the most ideal approach to expand the viability, productivity and inclusion of the product testing. When robotized tests are made they can undoubtedly be rehashed and they can be stretched out to perform assignments unimaginable with manual testing.

\section{Why Automated Testing?}

Manual programming testing is performed by a human sitting before a PC cautiously experiencing application screens, attempting different use and info blends, contrasting the outcomes with the normal conduct and recording their perceptions. Manual tests are rehashed frequently during improvement cycles for source code changes and different circumstances like numerous working situations and equipment setups.

A mechanized testing apparatus can playback pre-recorded and predefined activities contrast the outcomes with the normal conduct and report the achievement or disappointment of these manual tests to a test engineer. When mechanized tests are made they can without much of a stretch be rehashed and they can be reached out to perform undertakings unimaginable with manual testing. 


\section{Advantages of Automation Testing}

- $\quad$ Automated Testing sets aside time and cash.

- $\quad 70 \%$ faster than the manual testing.

- $\quad$ Reliable in results.

- $\quad$ Ensures consistency.

- Increases Efficiency.

- Human intervention is not required while execution.

\section{DIFFERENT METHODS USED FOR AUTOMATION TESTING}

\section{Selenium}

Selenium is a free (open-source) computerized testing system used to approve web applications across various programs and stages. Selenium is a valuable device since it isn't just open source yet in addition a convenient programming testing system for web applications that help various languages like Java, C\#, Ruby, Python. Picking the correct language relies upon the application under test, the supporting network, accessible test robotization systems, ease of use, class, and obviously, consistent form combination. Testing done utilizing the Selenium instrument is typically alluded to as Selenium Testing.

Other reasons behind Selenium's ever growing popularity are:

- Test contents can be written in any of these programming dialects like Java, Python, C\#, PHP, Ruby, Perl and .Net.

- $\quad$ Tests can be done in any of these OS: Windows, Mac or Linux.

- Tests can be completed utilizing any program: Mozilla Firefox, Internet Explorer, Google Chrome, Safari or Opera.

- It can be coordinated with instruments, for example, Test NG and JUnit for overseeing experiments and creating reports.

\section{Pro's}

- $\quad$ Open source, free to use and free of charge.

- Can run tests across various programs.

- $\quad$ Supports different working frameworks.

- $\quad$ Can execute tests in equal.

\section{Con's}

- Can just test web applications.

- $\quad$ Automates at a slower rate since it doesn't have a local IDE and just outsider IDE can be utilized for advancement. 
- Data-driven testing is progressively bulky since selenium depend on the writing computer programs language's abilities for setting esteems for the test information.

- Has no local ability to send out runtime information onto outside configurations.

- Parameterization should be possible by means of programming yet is hard to actualize.

- No local help to create test/bug reports.

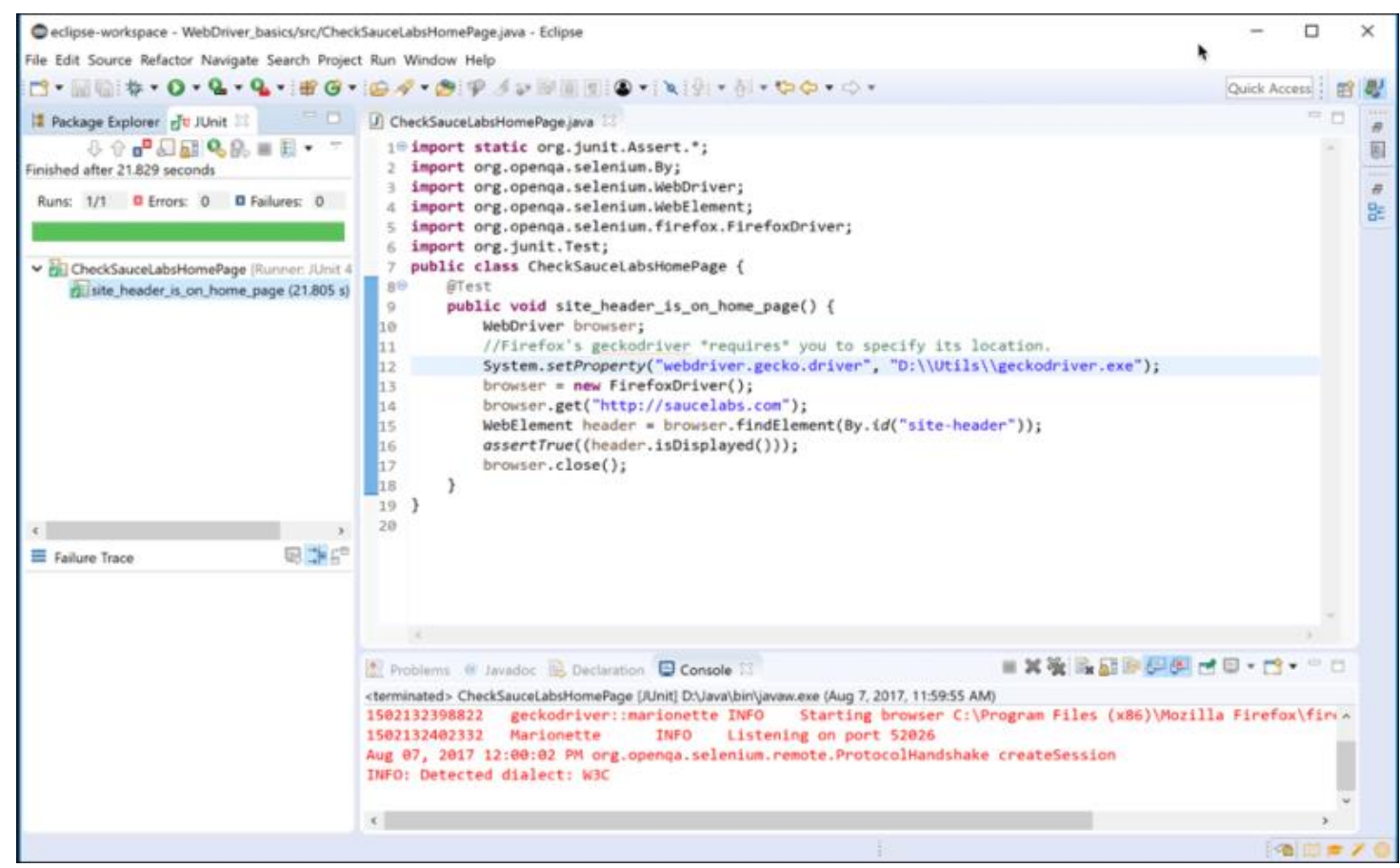

Figure 1: Result of Selenium Framework.

The above screenshot describes or explains about the result of using selenium in automation testing.

\section{Selenium with TestNG}

TestNG in Selenium is a Java testing structure, propelled by $\mathbf{J}$ Unit and $\mathbf{N}$ Unit. It conquers the limitations and burdens of $\mathbf{J}$ Unit. Its whole new arrangement of properties makes TestNG all the more impressive and simple to utilize. The addition 'NG' represents Next Generation, meaning the new functionalities that TestNG brings to the table. From basic unit testing to complex incorporated testing, it is intended to rearrange all our testing prerequisites, for example, practical testing, relapse, start to finish testing, and that's just the beginning.

It is architected to rearrange an expansive scope of testing needs beginning from unit testing to incorporated framework testing. At first, both J Unit and TestNG were structured exclusively for unit testing. TestNG is propelled by $\mathbf{J}$ Unit Java stage and N Unit .NET stage, and some new functionality was presented in TestNG, making it more remarkable and simple to use than the $\mathrm{J}$ Unit testing system.

\section{Pro's}

- TestNG Annotations are utilized to make experiments without any problem.

- Test cases can be 'assembled', 'organized' and 'executed' all the more effectively. It supports parameterization.. 
- It supports parallel execution.

- In TestNG, there is no need to state @ AfterClass and @ BeforeClass in a project, which is present in J Unit.

\section{Con's}

- For using TestNG one should have the knowledge of Java or Maven/ANT, loggers and more libraries (depending on the need)

- For writing scripts its takes time to write when compared with Robot Framework.

- Logs are not generated as compared to Robot Framework.

- Failure screenshots are generated as default unless a separate logic is written.

\begin{tabular}{|c|c|c|c|c|c|c|}
\hline Test & \# Passe & d \# Skipped & \# Retried \# Failed & Time (ms) & Included Groups & Excluded Groups \\
\hline \multicolumn{7}{|c|}{ Default suite } \\
\hline Default test & & 1 & 0 & 141 & & \\
\hline Class & & Method & Start & Time $(\mathrm{ms})$ & & \\
\hline \multicolumn{5}{|c|}{ Default suite } & & \\
\hline \multicolumn{5}{|c|}{ Default test - failed } & & \\
\hline Sample.Sam & bleTest & estMethodTwo & 1589123458779 & 20 & & \\
\hline \multicolumn{5}{|c|}{ Default test - skipped } & & \\
\hline Sample.Sam & bleTest & estVlethodThree & e 1589123458831 & 0 & & \\
\hline \multicolumn{5}{|c|}{ Default test - passed } & & \\
\hline Sample.Sam & leTest & est. Sethod0ne & 1589123458821 & 3 & & \\
\hline
\end{tabular}

Figure 2: Result of Selenium with TestNG Framework.

\section{Cucumber}

In the product business, a Behavior-Driven Development (BDD) device, Cucumber, has been generally utilized. Typically item examiners, designers, and analyzers physically compose BDD test situations that depict framework practices. Analyzers compose usage for the BDD situations by hand and execute the Cucumber tests. BDD approach is utilized in significant level testing, for example, framework testing or User Acceptance Testing (UAT), yet not in low-level testing, for example, unit testing. Cucumber gives a mapping component with the goal that analyzers can compose test code to guide to each unmistakable BDD situation step.

\section{Pro's}

- Cucumber gives straightforwardness about what test situations are secured and how the test situations are mapped to executable tests.

- It likewise gives straightforwardness among item examiners, designers and analyzers.

- Product investigators, who are by and large non-specialized individuals, can without much of a stretch comprehend what framework practices BDD situations spread.

- Easy for everybody to comprehend whether tests are accurately executed. 


\section{Con's}

- Test situation's are normally powerless and don't cover muddled cases.

- Practitioners don't have a measurement to quantify test inclusion.

- When a framework conduct changes, professionals need to physically change all events identified with that conduct in the test situations.

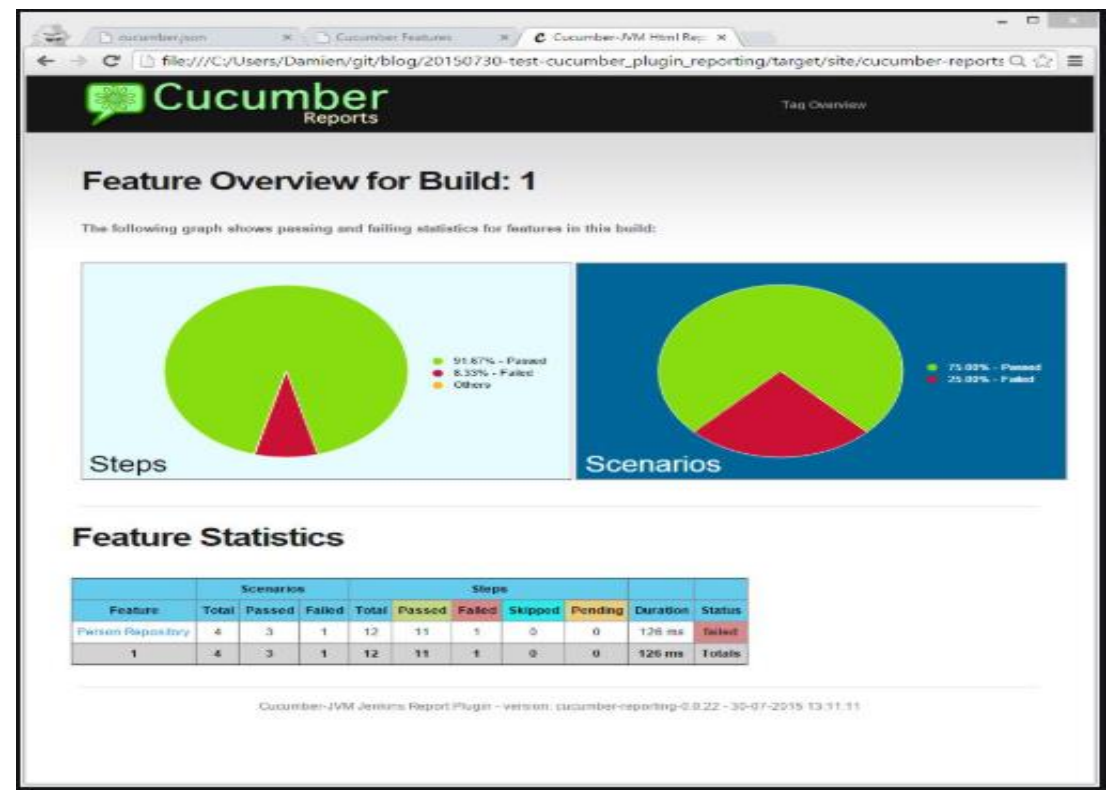

Figure 3: Detailed Report of Cucumber Framework.

\section{OVERVIEW ON ROBOT FRAMEWORK}

Robot Framework is a conventional test computerization structure for acknowledgment testing and acknowledgment testdriven turn of events. Robot Framework is created by Nokia Siemens correspondence innovation restricted organization. It has simple to-utilize even test information linguistic structure and uses the catchphrase driven testing and conduct driven methodology. Its testing capacities can be reached out by test libraries executed either with Python or Java, and clients can make new catchphrases from existing ones utilizing a similar grammar that is utilized for making experiments. The Framework presents the computerized testing, not exclusively would it be able to improve the testing proficiency, lessen programming relapse testing overhead, yet in addition is anything but difficult to utilize. It likewise gives Python or Java test library and different capacities.

Catchphrases are partitioned into more significant level client watchwords and lower-level library catchphrases. The client catchphrases are worked by making mixes from the watchwords introduced in the catchphrase libraries. The accessible catchphrases of Robot Framework are characterized in libraries. There are two kinds of libraries: standard and outside. The standard libraries are disseminated with Robot Framework and the outside libraries are discharged in discrete bundles:

Standard Libraries: these are the libraries that are bundled with the framework or it is also called as built-in libraries. Some of the standard libraries are: 
- BuiltIn: provides a set of common generic keywords. This comes with the imports automatically or available with imports.

- Operating System: provides operating framework errands to be performed in the system that are necessary for test cases.

- $\quad$ Process: Library for running procedures in the framework.

- Screenshot: Provides catchphrases to catch screen captures of the work area.

- XML: Library for creating, altering and confirming XML records.

External Libraries: these are the libraries that with the framework or it is also called as built-in libraries. Some of the external libraries are:

- Database Library: Python based library for database testing. Works with any Python translator.

- $\quad$ Sikuli Library: Sikuli Robot Framework Library provides keywords to test UI through Sikulix.

- AutoIt Library: AutoIt library is used to test the Windows GUI where Autolt freeware tool is used as a driver.

- $\quad$ Selenium Library: Selenium is used as Web testing library.

- $\quad$ SSH Library: SSH library is utilized to execute orders on remote machines over a SSH association.

\section{Robot Framework Architecture:}

Robot Framework is computerized structure dependent on catchphrase driven. The watchword driven innovation permits administrations, information and contents autonomous to one another in the computerized testing.

Robot Framework is an adaptable, which its application and strategy is free of one another. Simultaneously, it is a lot of mechanized testing instrument. It has a particular design appeared in figure and every one of modules has its own capacity as follows:

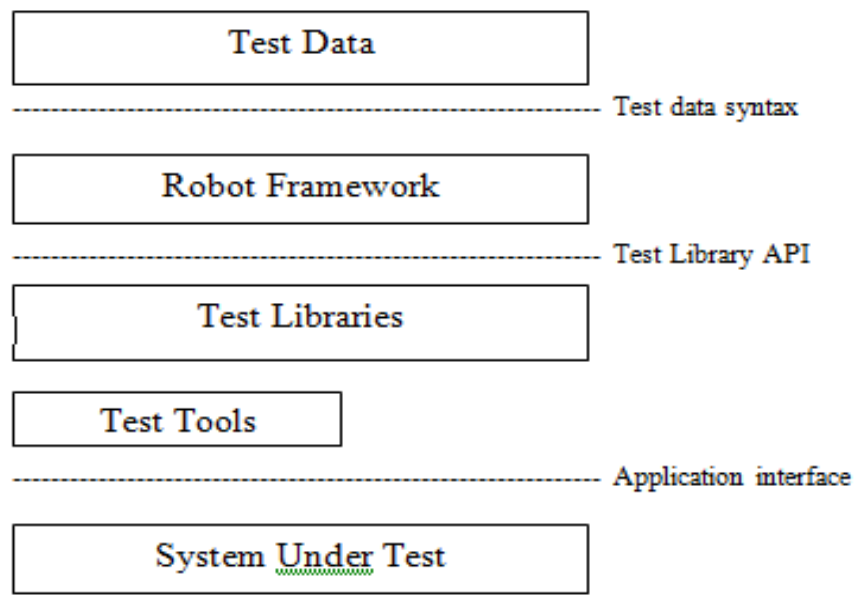

Figure 4: Robot Framework Architecture. 
Test Data: that is a Tabular structure which is anything but difficult to alter. When to begin the Robot Framework, it will utilize the test information to run experiments, and creates logs and reports. The center of the structure is unusual for the diverse sort measure frameworks, and is intelligent with the measure frameworks through the Test Libraries. The test library can legitimately use as the application program interface or lower levels of testing devices as driving.

Robot Framework: It must be run on Python or Jython, the deliberate framework must arrangement by Python or Jython, on the grounds that Robot Framework source code is composed by Python language, and a few frameworks must be in the Python to run, so we propose introducing Python to utilize Robot Framework. We can utilize the order to begin system and afterward start the test information document, execute experiment.

Test Libraries: It is made out of two sections, with BuiltIn Library Robot structure, just as the R\&D work force as indicated by the test prerequisites, in light of the python language can be reached out to Extend Library.

System under Test: It is the item to be tried.

\section{Advantages of Robot Framework}

- It is platform and application independent.

- Can merge xml files of different test suites to generate one single report.

- Once created keywords can be reused within the test case or a project and can be used in the other test suite also.

- Results are generated in the form of reports and logs in HTML format.

- $\quad$ Provides labelling to arrange and choose experiments to be executed.

- Provides experiment and test-suite - level arrangement and teardown.

\section{Disadvantages of Robot Framework}

- Parallel execution is not possible.

- If-else statements are not allowed.

- $\quad$ No nested loops.

- It is very hard to find who knows or who has worked on Robot framework compared to java.

- Failures brought about by experiments themselves or by catchphrases utilized can here and there be difficult to troubleshoot.

\section{REPORTS OF ROBOT FRAMEWORK}

Report is an archive that records information got from an examination of assessment in a sorted out way, depicts the ecological or working conditions, and shows the correlation of test results with test destinations. It justifies the testing efforts of the team as well as the steps \& techniques used by them by providing an insight into the whole process. 


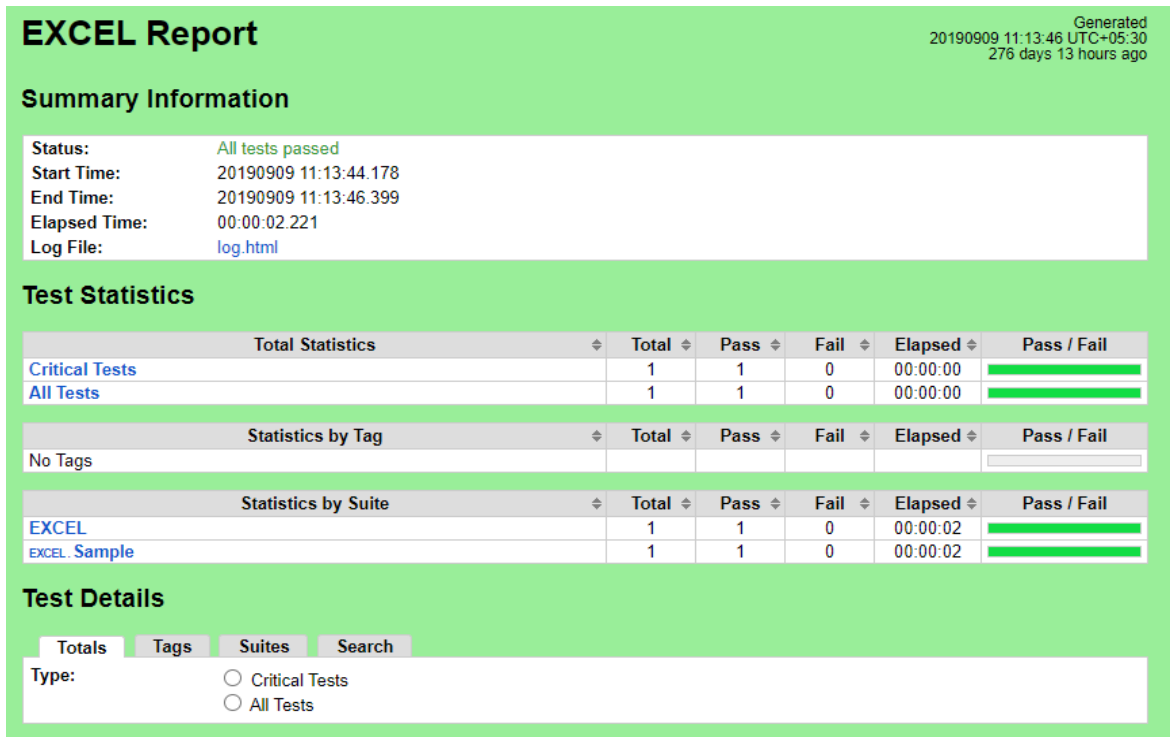

Figure 5: Pass Report of Robot Framework.

The above screenshot describes the pass report of the test case which indicates in green colour.

\begin{tabular}{|c|c|c|}
\hline \multicolumn{2}{|l|}{ - SUITE EXCEL } & \multirow[t]{5}{*}{00000.02221} \\
\hline Full Name: & EXCEL & \\
\hline Source: & C.Userskanyseaipse-workspoce EXCEL & \\
\hline Start/End/ Elapsed: & 20190909 11:13:44.178 / 201990999 11:13:46.399/ /00:00.022.221 & \\
\hline Status: & $\begin{array}{l}\text { latiocal lest } 1 \text { passed, } 0 \text { falled } \\
\text { ctest total, } 1 \text { passed, Ofalled }\end{array}$ & \\
\hline \multicolumn{2}{|l|}{ - suite Sample } & \multirow[t]{5}{*}{$00.00: 12190$} \\
\hline Full Name: & EXCELSample & \\
\hline Source: & C:Userskayssedipse-workspoceEXXCElSample robot & \\
\hline Start / End / Elapsed: & 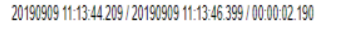 & \\
\hline Status: & $\begin{array}{l}1 \text { critcal test, } 1 \text { passed } 0 \text { falled } \\
1 \text { test total, } 1 \text { passed, } 0 \text { faled }\end{array}$ & \\
\hline \multicolumn{2}{|l|}{ - TEST Test2 } & \multirow[t]{4}{*}{00000000.062} \\
\hline Full Name: & EXCELSSample.Test2 & \\
\hline Start/ End / Elapsed: & 20190909 11:13:46.337/ $20190909911: 13.46 .399 / 00.00 .000062$ & \\
\hline Status: & PASS (cotical) & \\
\hline \multicolumn{2}{|c|}{ - rainsonopen the Excolsheet } & \multirow[t]{2}{*}{00000000.062} \\
\hline Stat/ End / Elapsed: & 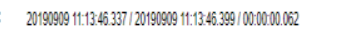 & \\
\hline \multicolumn{2}{|c|}{ 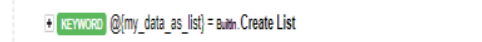 } & 00:00:00:000 \\
\hline \multicolumn{2}{|c|}{ 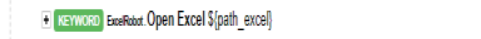 } & 00:00:00:015 \\
\hline \multicolumn{2}{|c|}{ 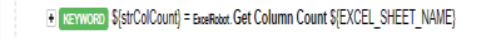 } & $00: 00: 00.016$ \\
\hline \multicolumn{2}{|c|}{ 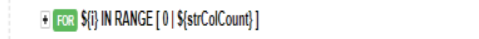 } & 00:00:00:031 \\
\hline \multicolumn{2}{|c|}{ 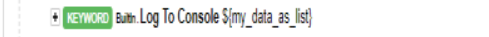 } & $00: 00: 00.000$ \\
\hline
\end{tabular}

Figure 6: Detailed Pass Report of Robot Framework.

The above screenshot describes the detailed information about test case execution and its timings. 


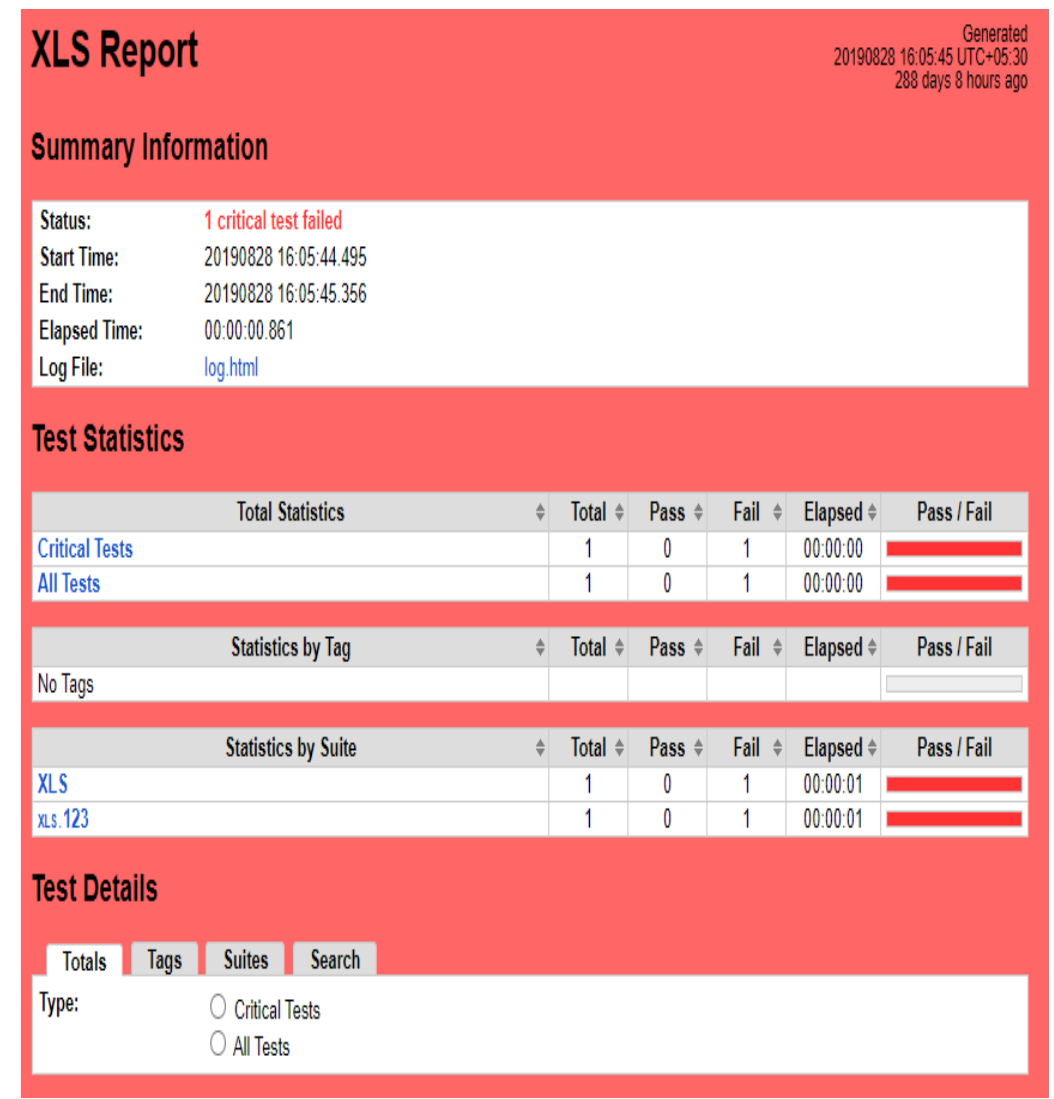

Figure 7: Fail Report of Robot Framework.

The above screenshot describes the fail report of the test case which indicates in red colour and it is easily understandable just by the colour without knowing the details.

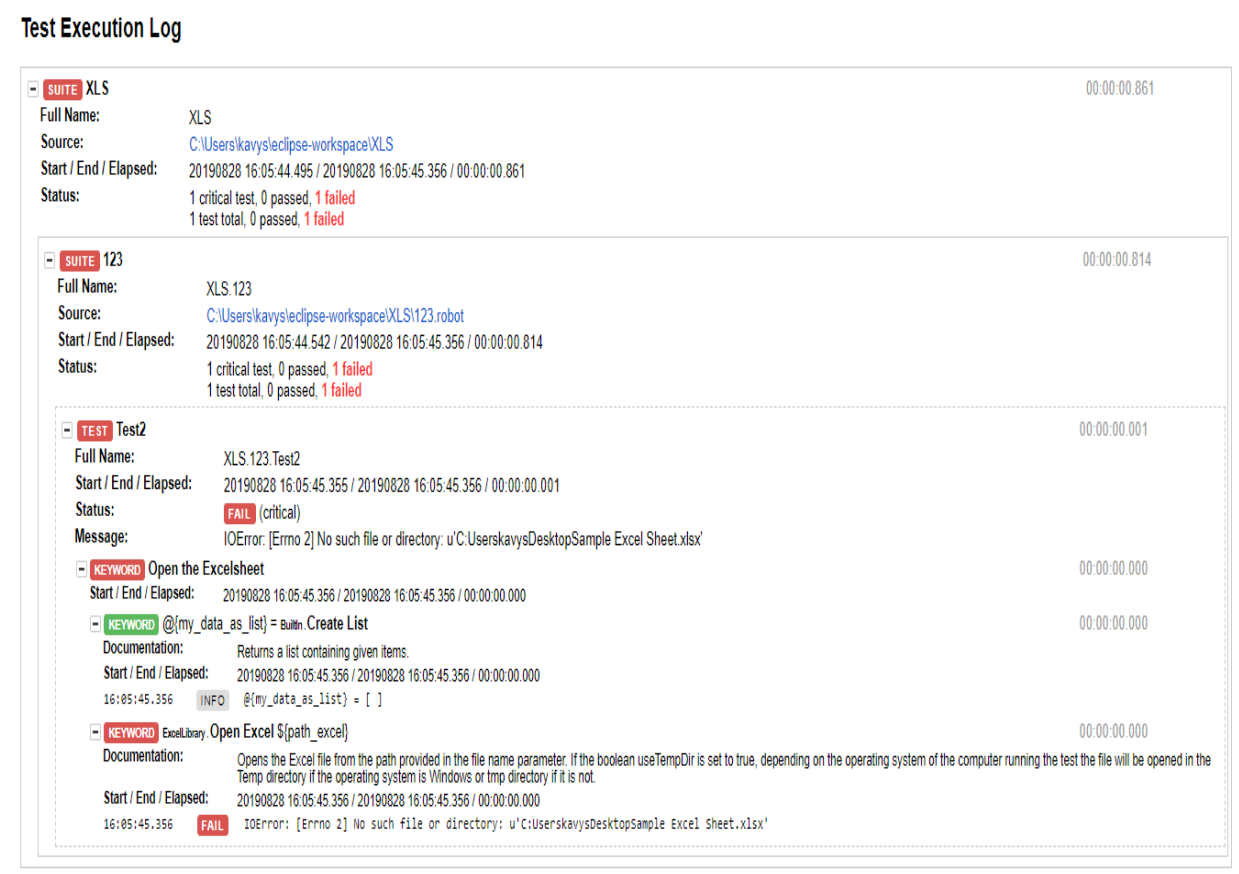

Figure 8: Detailed Report of Fail Report with Screenshot. 
The above screenshot describes the test execution log which appears in log part of the report. This clearly tells which statement or line has failed to execute with the screenshot. This is the added advantage of the robot framework.

\section{CONCLUSIONS}

This paper analyses that robot framework is best among the discussed methods for automation testing. Robot Framework which depends on mechanized testing system. It is likewise a keyword driven computerized test table casing and it will make the procedure and the board increasingly normalized. The after effects of the analysis demonstrate that Robot Framework has critical points of interest over most prevalently utilized strategies as a result of its it is easy to learn and ground-breaking.

It is beneficial for regression testing, increases the code utilization rate, reduces the human intervention and therefore increases the efficiency of testing. Robot framework's reports are more efficient than any other automation tools because of its easy-to-read capability. Its added advantages are reusable code, rich libraries, command line execution, selected test case can be executed by tagging and many more. These all advantages make Robot Framework a secure and reliable automation framework.

\section{REFERENCES}

1. Liu Jian-Ping, Liu Juan-Juan, Wang Dong-Long: Application Analysis of Automated Testing Framework Based on Robot; 2012 Third International Conference on Networking and Distributed Computing, Comibatore, Feb. 25 - 27, 2015.

2. Qiu Na, Du Huaichang; Extension and Application Based on Robot Testing Framework; 2015 Third International Conference on Software Testing; Tamilnadu, Dec. 2-4, 2015.

3. N. Haller, C. Metz, P. Nesser, and M. Straw, “A OneTime Password System,” Internet Engineering Task Force Request For Comments 2289, IETF Website, 1998.

4. Paruchuri Ramya, Vemuri Sindhura, P Vidya Sagar; Testing using Selenium Web Driver; 2017 International Conference on Software Testing; Malaysia, Feb 15-17, 2017

5. Antawan Holmes, Marc Kellogg; “Automating Functional Tests Using Selenium”, 2006 Third International Conference on Computer Applications; Singapore, May 28-31, 2006.

6. K. V. Arya, Hemdutt Verma; "Keyword Driven Automated Testing Framework for Web Application”; 2018 International Conference on Computer applications and networks; Pune; June 5-8, 2018

7. Rakesh Kumar Lenka, Kabita Manjari Nayak, Sarthak Padhi; “Automated Testing Tool: QTP”; 2018 International Conference on Advances in Computing, Communication and Control and Networking; Malaysia, 8-10 May 2018.

8. Sandeep Sivanandan, Yogeesha C.B; "Agile Development Cycle: Approach to Design an Effective Model Based Testing with Behaviour Driven Automation Framework"; 2018 International Conference on Computer networks; Bangalore, 8-10 May 2018 .

9. Nan Li, Anthony Escalona, Tariq Kamal; "Skyfire: Model-Based Testing With Cucumber"; 2016 IEEE International Conference on Software Testing, Verification and Validation; 28-31 January 2016.

10. Li Bobo, Zhan zhiliang, “Applications of Robot Framework in distributed control system testing," Industrial control computer, vol. 27, pp. 37-38,41,2014. 
11. Tuomas Pajunen, Tommi Takala, Mika Katara: Model-Based Testing with a General Purpose Keyword-Driven Test Automation Framework; 2011 Fourth international Conference on Software Testing, Verification and Validation Workshops, New York, USA, 1-3 Aug. 2011.

12. Sahu, Supriya, and B B Choudhury. "Cycle Time Optimized Path Planning for Industrial Robot using Robomaster." International Journal of Mechanical and Production Engineering Research and Development (IJMPERD) 7.5 (2017):95-102.

13. Aruna, Chittineni, and R. Siva Ram Prasad. "Development of an Automated Framework to Resolve Software Testing Issues." International Journal of Computer Science Engineering and Information Technology Research (IJCSEITR) 4.2 (2014):259276.

14. Rasal, S P, Harsha Ingale, and V S Shelar. "Object Tracking System Using Image Processing." International Journal of Computer Science Engineering and Information Technology Research (IJCSEITR) 3.4 (2013):137-142.

15. Bhasin, Harsh, ANKUSH GOYAL, and DEEPIKA GOYAL. "Software Architecture Based Regression Testing Implementation." International Journal of Computer Science and Engineering (IJCSE) 2.3 (2013):1-4. 